\title{
Description of two new species of Physopyxis and redescription of $P$. lyra (Siluriformes: Doradidae)
}

\author{
Leandro M. Sousa and Lúcia H. Rapp Py-Daniel
}

\begin{abstract}
The genus and species Physopyxis lyra was originally described based on only one very distinctive specimen. Analysis of a larger number of specimens provided a more precise characterization of the genus and the type species. Additionally, two new species are described based on material already available in museums and new collections. Physopyxis lyra is characterized by having a well developed coracoid process with divergent tips, and only one series of spines on the lateral plates. Physopyxis ananas, new species, has a thin coracoid process, convergent, and more than one series of spines on the lateral plates. Physopyxis cristata, new species, is a slender species, characterized by an incomplete series of lateral plates and the presence of a middorsal series of spines formed by the tips of the vertebral neural spines. The poorly known geographic distribution of the genus Physopyxis is vastly enlarged to include various drainages of the Amazon and Essequibo basins.

Physopyxis lyra foi originalmente descrita com base em apenas um exemplar de características muito peculiares. Análises de um grande número de exemplares possibilitou uma caracterização mais precisa do gênero e da espécie tipo. Adicionalmente, duas novas espécies são descritas com base em material disponível em museus e em exemplares coletados recentemente. Physopyxis lyra é caracterizado por possuir um processo coracóide muito desenvolvido com as extremidades voltadas para fora, e por apresentar placas laterais com apenas uma fileira de espinhos. Physopyxis ananas, espécie nova, possui processo coracóide estreito e ligeiramente convergente, com mais de uma fileira de espinhos ao longo das placas laterais. Physopyxis cristata, espécie nova, é uma espécie menos robusta, caracterizada por possuir linha lateral incompleta com placas pouco desenvolvidas e presença de uma fileira mediana dorsal de espinhos, formada pela extremidade dos espinhos neurais das vértebras. A distribuição geográfica do gênero Physopyxis, muito pouco conhecida até então, é bastante ampliada, incluindo agora diferentes drenagens da bacia Amazônica e do Essequibo.
\end{abstract}

Key words: Plates ornamentation, Incomplete lateral line, Taxonomy, Thorny catfish.

\section{Introduction}

The Doradidae is a South American catfish family easily recognized by the presence of spiny bony plates along the lateral line. Currently, the family comprises 72 valid species in 30 genera (Sabaj \& Ferraris, 2003). The monotypic genus Physopyxis Cope, 1871, is a remarkable minute doradid with adults not known to exceed $31 \mathrm{~mm}$ SL. Physopyxis lyra has always been easily recognized. Cope's original description, however, depicts some morphological discrepancies with the unique type and other $P$. lyra specimens. For instance, Cope diagnosed Physopyxis lyra by the absence of an adipose fin, and his statement was accepted by Eigenmann (1925) and Burgess (1989). However, the type specimen is in very poor condition (i.e., completely desiccated) and it is difficult to determine whether an adipose fin was originally present. The vast majority of the specimens of $P$. lyra examined in this study do show an adipose fin.

Higuchi (1992) pointed out that there were some discrepancies between the illustration and the text in Cope's (1872) expanded description of $P$. lyra (Fig. 1). The illustration shows an unnamed structure connecting the posterior nuchal plate to the base of the pelvic fin. This structure is neither present in the holotype nor in any of the examined specimens. The illustration of the anal fin in $P$. lyra also is exceedingly quadrangular, different from that of all other examined specimens.

Material recently collected and assigned to $P$. lyra has considerably expanded the geographic distribution of the taxon and raised some doubts about the monotypy of the genus. In this paper, we present a taxonomic review of Physopyxis, with a redescription of $P$. lyra and descriptions of two new species, and comment on morphological variation observed in the group.

Instituto Nacional de Pesquisas da Amazônia - INPA, Av. André Araújo, 2936, Petrópolis, 69011-830 Manaus, AM, Brazil. rapp@inpa.gov.br, leandro.m.sousa@gmail.com 

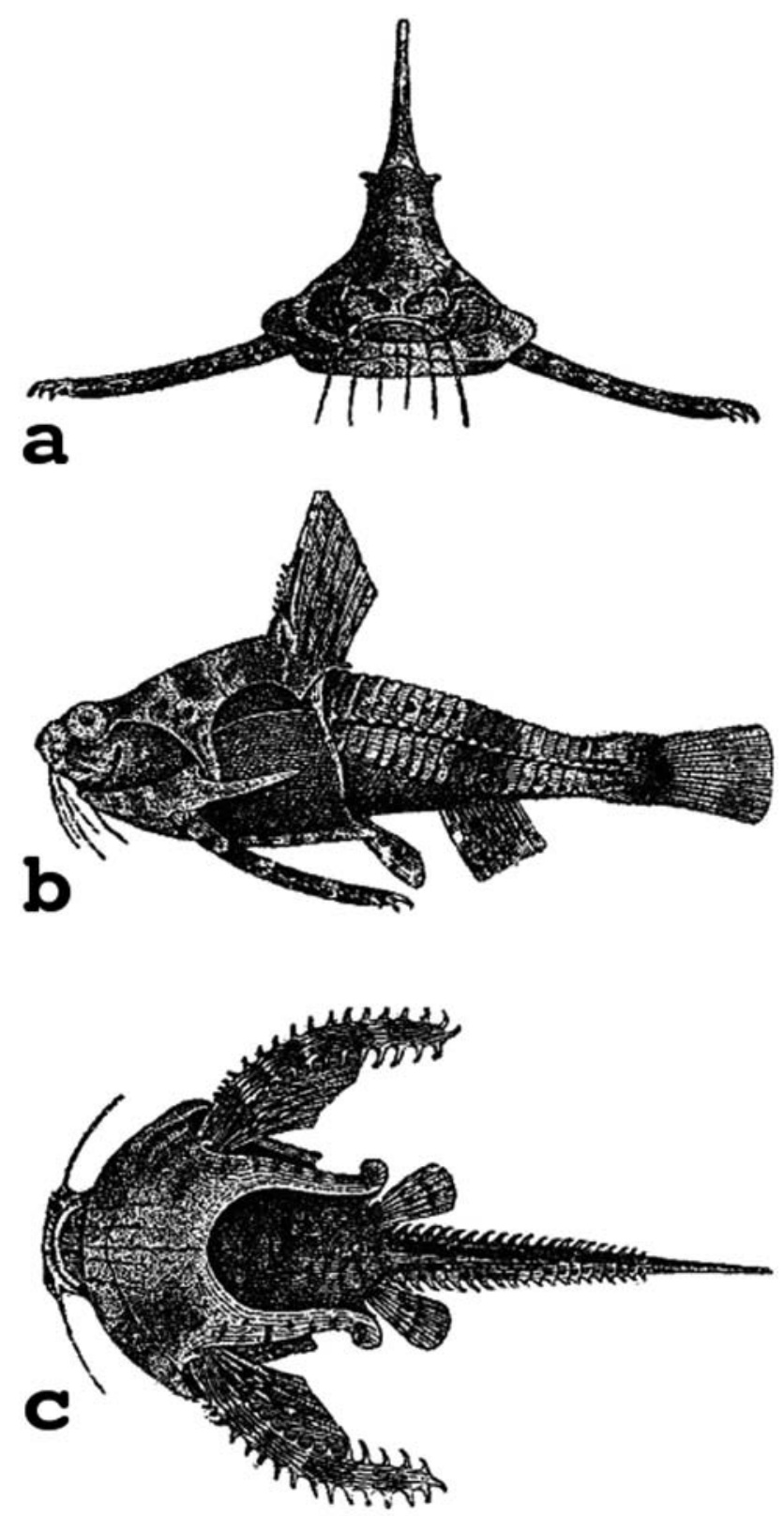

Fig. 1. Physopyxis lyra. Ilustration from original description by Cope (1872). a. anterior view; b. lateral view; c. ventral view.

\section{Material and Methods}

We examined 2250, and measured 346, specimens of Physopyxis. Most measurements and counts follow Higuchi et al. (1990) and Sabaj (2002), and measurements were taken to $0.01 \mathrm{~mm}$ with a digital caliper under a stereomicroscope. Additional measurements are: lateral plate depth - depth of 5 th plate (modified from Sabaj, 2002); superior wing depth - depth of superior wing of 5 th plate, taken from the dorsal-most exposed contour of plate to point of lateral line spine (modified from Sabaj, 2002); inferior wing depth - depth of inferior wing of 5th plate, taken from the ventral-most exposed contour of plate to point of lateral line spine; body depth at 5 th plate - greatest body depth at 5th plate; scapular girdle width - greatest body width at cleithra; postcleithral (humeral) process length - length from anteriormost point of contact between supracleithrum and cleithrum to tip of postcleithral (humeral) process; coracoid process length - length from posteriormost border of pectoralfin point of insertion to tip of the coracoid process; pectoral girdle mesial suture - greatest longitudinal measure of the mesial suture between cleithra and coracoid; distance between coracoid process tips - shortest ventral distance between the tips of coracoid processes; coracoid width - width at external most sides of coracoids at the level of origin of the pectoral fin. Measurements were taken, whenever possible, on the left side of the body and were treated as percents of the standard length (SL). Cephalic measures, such as head depth, snout length, orbital diameter, interorbital distance and mouth width were treated as percents of the head length (HL).

Caudal-fin ray counts follow Lundberg \& Baskin (1969). In double-stained specimens examined, principal caudal-fin rays (those that articulate with the parhypural and hypurals) usually include one long unbranched ray in the upper lobe and all the subsequent branched rays. However, in some specimens it was observed that branched rays can also be found on the lower lobe ventral to the hypural plate. In intact specimens it is not possible to verify of the position of caudal-fin rays without clearing and staining or $\mathrm{x}$-raying. Therefore, principal caudal rays were counted as the first long unbranched fin ray plus all following branched rays. In the text, these counts are represented by 8,4 or 8,3 or 7,4 (= upper-lobe fin rays and lower-lobe fin rays).

Some measurements could not be taken on the holotype of Physopyxis lyra due to its fragile condition. The pectoral, anal and caudal fin-ray counts of the holotype are those found in Cope's original description. Clearing and double-staining procedures follow Taylor \& Van Dyke (1985). Illustrations were made under a camera lucida attached to a stereomicroscope. Specimens of Amblydoras, a taxon closely related to Physopyxis (Higuchi, 1992), were used for anatomical and skeletal comparisons. Acronyms follow Leviton et al. (1985) with addition of UNAP (Universidad Nacional de la Amazonía Peruana).

\section{Results}

\section{Physopyxis Cope, 1871}

Physopyxis Cope, 1871: 112; described in detail in Cope, 1872: 273; type-species, Physopyxis lyra Cope, 1871, by monotypy.

Diagnosis. Physopyxis can be distinguished from the other Doradidae by the miniature size (adults less than $31 \mathrm{~mm} \mathrm{SL}$ ) and a unique combination of additional features: extreme development of the exposed pectoral girdle; well developed coracoid process longer than the postcleithral process; anterior plate of the nuchal shield absent; dorsal spine serrated 
only along the basal portion of its anterior margin; two infraorbital bones in young that become fused into a single plate in adult specimens; pectoral spine reaching to base of anal fin.

\section{Key for species of Physopyxis.}

1. Lateral line incomplete, with 5 to 12 lateral plates, each with one retrorse spine; a series of small spines along dorsal midline formed by the external expansion of the vertebral neural spines; adipose fin present (Fig. 2a) .... P. cristata (rio Negro basin)

1'. Lateral line complete; lateral plates well developed to caudal fin and meeting dorsally in larger specimens; adipose fin present or absent (Figs. 2b-c)
2. Lateral plates with only one series of spines; superior wing of lateral plates deeper than inferior wing (Fig. 2b); coracoid processes with posterior tip enlarged, knob-like and diverging laterally (Fig. 2e); adipose fin usually present

P. lyra

(Amazon basin in Peru and Brazil)

2 '. Lateral plates with two or more series of spines; depth of superior wing of lateral plates equal to or less than that of inferior wing (Fig. 2c); coracoid processes narrow and converging toward central axis of the body (Fig. 2d); adipose fin usually absent

P. ananas

(widely distributed in Amazon and Essequibo basins)

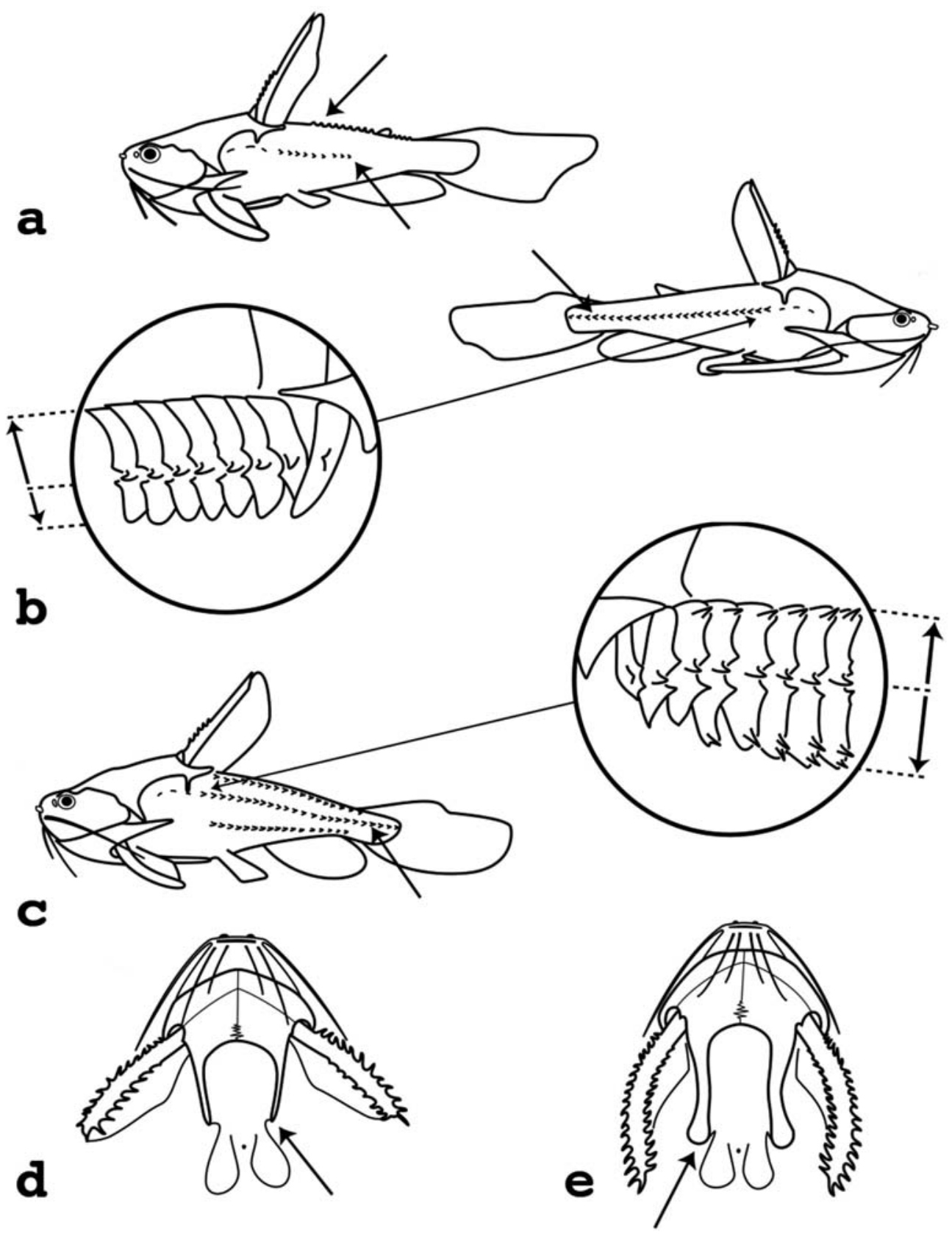

Fig. 2. Diagnostic characters of the three species of Physopyxis. a. Physopyxis cristata; b. Physopyxis lyra; c. Physopyxis ananas; d. ventral view of scapular girdle in $P$. ananas and $P$. cristata; e. ventral view of scapular girdle in $P$. lyra. 


\section{Physopyxis lyra Cope}

Figs. 3-4

Physopyxis lyra Cope, 1871: 112; described in detail and illustrated in Cope, 1872: 273 pl. 5 fig. 1a-c, type locality: rio Ampyiacu, Peru (stated in Cope, 1872: 275 as rio Ambyiacu (sic), Equador).

Material examined $(\mathbf{N}=\mathbf{1 6 6 2})$. ANSP 8282 (holotype, $35.0 \mathrm{~mm}$ TL), río Ampyiacu, Peru. Other specimens: Brazil: Amazonas: AUM 44217 (10, not measured), igarapé Juá Grande, lago Amanã, rio Japurá, rio Solimões basin. INPA $77(1,21.83 \mathrm{~mm} \mathrm{SL})$, rio Aripuanã, rio Madeira basin. INPA 15470 (21 of 354, 13.37-23.05 mm SL), igarapé Caititu, rio Uatumã, Balbina. INPA 20321 (4 c\&s, not measured), rio Tefé, rio Solimões basin. INPA 21922 (1, $14.81 \mathrm{~mm} \mathrm{SL})$, rio Padauari, rio Negro basin. INPA 21926 (34 of 949, 15.25-30.63 mm SL), igarapé Juá Grande, lago Amanã, rio Japurá, rio Solimões basin. MPEG 9718 (15, not measured), same data as INPA 21926. MZUSP 88066 (15, not measured), same data as INPA 21926.

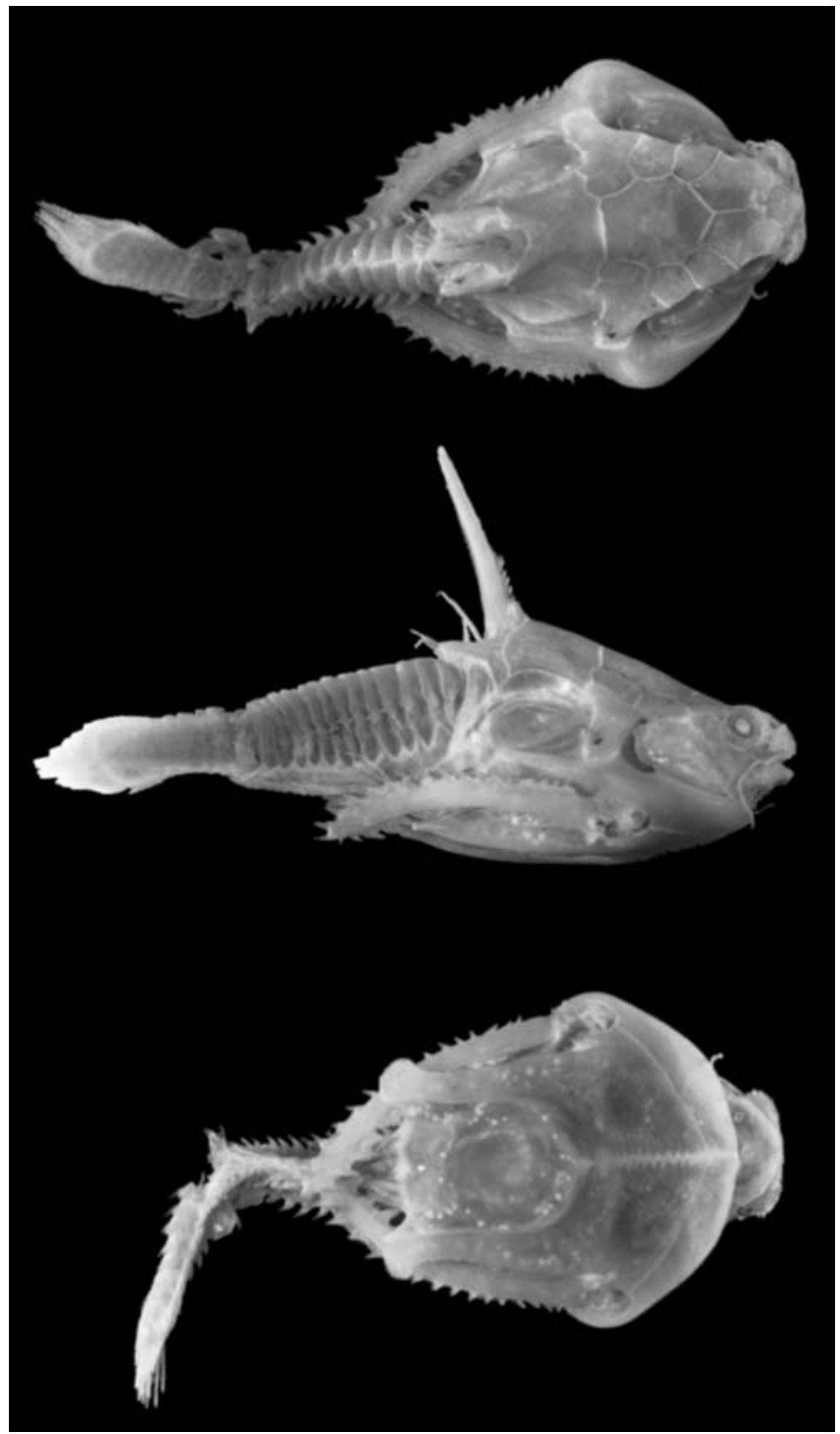

Fig. 3. Physopyxis lyra Cope, 1871 , holotype. ANSP 8282 (35.0 mm TL), río Ampyiacu, Peru. Photo by Mark Sabaj.
Mato Grosso: INPA 25029 (5, 19.1-20.91 mm SL), rio Guaporé. MZUSP 37670 (1, $21.26 \mathrm{~mm} \mathrm{SL})$, rio Guaporé, ferry boat harbour. Rondônia: INPA 11318 (1, not measured), rio Guaporé, Costa Marques county. INPA 21920 (3, 16.37-23.94 mm SL), rio Cautário, rio Guaporé basin. INPA $21921(6,18.53-24.66 \mathrm{~mm} \mathrm{SL})$, rio Jatobá, rio Guaporé basin. INPA $21923(1,18.81 \mathrm{~mm} \mathrm{SL})$, rio Novo, Pacaás Novos (1056'24"S, 65'56'24”'W). INPA 21924 (1, $20.03 \mathrm{~mm} \mathrm{SL}$ ),

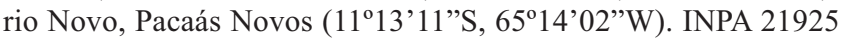
(9, 18.63-27.67 mm SL), rio Cautário, rio Guaporé basin. INPA 21927 (14, 12.62-26.28 mm SL), rio Cautário, rio Guaporé basin. Peru: Loreto: ANSP 116359 (1 c\&s, not measured), río Nanay, vicinity Iquitos. ANSP $149338(9,18.17-26.62 \mathrm{~mm} \mathrm{SL})$, río Nanay.

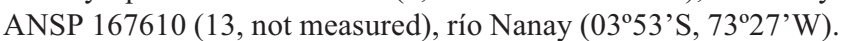
ANSP 167615 (53, 16.14-30.34 mm SL) (2 c\&s, not measured), río Nanay $\left(03^{\circ} 45^{\prime} \mathrm{S}, 7^{\circ} 17^{\prime} \mathrm{W}\right)$. ANSP $167619(15,14.34-29.84 \mathrm{~mm}$ $\mathrm{SL})$, río Nanay $\left(03^{\circ} 45^{\prime} \mathrm{S}, 73^{\circ} 17^{\prime} \mathrm{W}\right)$. ANSP $174930(1,25.78 \mathrm{~mm}$ $\mathrm{SL})$, río Ucayali, río Nanay $\left(05^{\circ} 00^{\prime} \mathrm{S}, 7^{\circ} 38^{\prime} \mathrm{W}\right)$. ANSP 178442 (12, 20.04-29.00 mm SL), río Nanay ( $03^{\circ} 45^{\prime} 09^{\prime}$ 'S, $\left.73^{\circ} 17^{\prime} 00^{\prime} \mathrm{W}\right)$. ANSP 179872 (151, not measured), río Nanay ( $\left.03^{\circ} 46^{\prime} 45^{\prime \prime} \mathrm{S}, 73^{\circ} 22^{\prime} 06^{\prime} \mathrm{W}\right)$. ANSP 179873 (17, not measured), río Nanay, Pampa Chica village $\left(03^{\circ} 45^{\prime} 09^{\prime \prime} \mathrm{S}, 73^{\circ} 17^{\prime} 00^{\prime} \mathrm{W}\right)$. MZUSP 26783 (1, $\left.23.26 \mathrm{~mm} \mathrm{SL}\right)$, Jenaro Herrera.

Diagnosis. Physopyxis lyra differs from the other species of the genus by possessing a single row of spines on the lateral plates and a strong scapular girdle with long, broad coracoid processes having distal tips enlarged and divergent.

Description. Morphometric data summarized in Table 1. Largest specimen examined measures $30.63 \mathrm{~mm}$ SL (39.07 mm TL) representing largest known specimen. Body short and deep with greatest body depth at origin of dorsal fin (body depth $27-35.6 \%$ of SL). Head and scapular girdle strongly ossified, exposed. Top of head and nuchal plates roof shaped, with bony ornamentation organized in shallow grooves concentrated along nuchal shield crest and borders. Single fontanel oval shaped. Eye anterior, orbital diameter approximately same size as fontanel. Anterior nostril tubular and placed near border of snout. Posterior nostril nearer to orbit than to snout. Snout short, its length twice orbital diameter. Lacrimal bone well developed, dorsal and ventral margins serrated with conspicuous spines. Mouth terminal. Barbels simple, long, covered with papillae along inferior surfaces. Maxillary barbel reaching to or surpassing tip of postcleithral process. Outer mental barbel reaching point of insertion of pectoral spine. Inner mental barbel shorter, approximately two thirds of length of outer mental barbel.

Scapular girdle extremely well developed anteriorly and ventrally, forming deep groove along its anterior contour. Cleithrum laterally expanded and visible from above, sculptured with shallow grooves up to postcleithral process. Postcleithral process relatively short, its tip reaching to or slightly surpassing vertical through origin of dorsal spine. Coracoid processes very long and broad (larger than postcleithral process), with expanded distal tip. Ventral surface of coracoid process ornamented with well-defined longitudinal pattern of parallel grooves and ridges along its entire length. At posterior tip of coracoid, grooves and ridges curve 
outwards following expansion of process.

Dorsal and pectoral spines strongly ossified. Dorsal spine pentagonal in cross-section with longitudinal groove along each lateral side, serrate along basal portion of anterior margin, posterior margin smooth. Pectoral spine well developed, depressed and curved, its tip usually reaching anal-fin origin. Two or three grooves on both upper and lower surfaces of pectoral spine. Strong hook-like teeth present along anterior and posterior margins of pectoral spine; basal most teeth (i.e., first five) along anterior margin directed toward base of spine, more distal teeth directed toward spine tip; all teeth along posterior margin directed toward pectoral-spine base.

Vast majority of observed specimens with 26 lateral plates, each with single posteriorly directed spine. Number of lateral plates 23-27, with few juvenile specimens (less than $16.37 \mathrm{~mm}$ SL) from Guaporé drainage lacking plates entirely (see Table

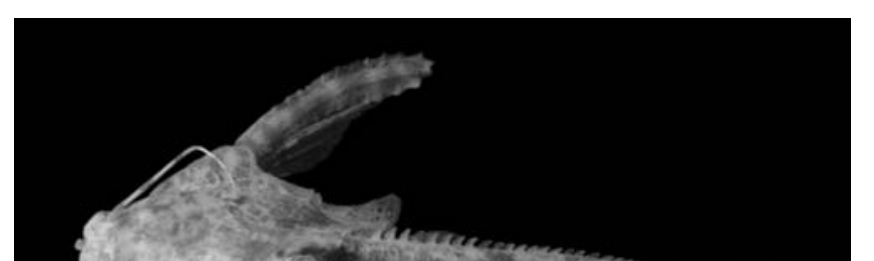


one that covers entire caudal peduncle. Barbels tan with brown transverse bands along its entire length. All fins similar in appearance, with dark transverse bands across rays and membranes separated by unpigmented interspaces. Dorsal and pectoral spines with unpigmented tips. Spines and rays with brown transverse bands. Ventral surface variably pigmented, light or dark, with chromatophores regularly spaced over abdomen and scapular bridge. Coloration in fresh specimens similar to above, but more conspicuous.

Distribution. Known from the rio Ampyiacu (and lowland portions of other tributaries to the upper Amazon in northeastern Peru) to the rio Uatumã, a left bank tributary to the Amazon in eastern Amazonas State, Brazil. No reliable records exist for the rio Negro basin (one specimen recorded from upper rio Negro may be incorrectly labeled) (Fig. 6).

Habitat. Physopyxis lyra is usually found in places with accumulated organic debris, like dense meshes of roots of floating macrophytes that are abundant in rivers with turbid water. Specimens also can be found among submersed leaf litter and among root mats of riparian plants, like Symmeria paniculata (Polygonaceae). Aquarium observations indicate that $P$. lyra is predominantly nocturnal, and spends most of day time sheltered among submersed root mats or buried in sand.

\section{Physopyxis ananas, new species \\ Fig. 5}

Holotype. INPA 24324 (13.43 mm SL), rio Jutaí, rio Solimões basin, Amazonas State, Brazil (02 $45^{\circ} 40^{\prime \prime}$ S, 66 $\left.47^{\circ} 59^{\prime \prime} \mathrm{W}\right)$, ProVárzea expedition, 7 Sep 2003.

Paratypes ( $\mathbf{N}=\mathbf{5 6 3})$. Brazil: Amazonas: ANSP 149342 (2, not measured), mouth of Tapauá, rio Purus. ANSP 180962 (10, not measured), igarapé Juá Grande, lago Amanã, rio Japurá, rio Solimões basin. INPA 6625 (10, 12.0-18.8 mm SL), igarapé do Santo Antônio, rio Negro basin, Santa Isabel do Rio Negro. INPA 13420 (6, 11.71-13.51 mm SL), rio Tarumã-mirim, rio Negro basin. INPA $18448(2,13.29-13.51 \mathrm{~mm} \mathrm{SL})$, rio Tarumãmirim, rio Negro basin. INPA 18450 (1, $13.05 \mathrm{~mm} \mathrm{SL})$, rio Marié. INPA 20197 (1, $11.05 \mathrm{~mm} \mathrm{SL}$ ), igarapé Zamula, rio Negro basin. INPA 20198 (3, 11.15-12.6 mm SL), rio Cuiuni, rio Negro basin. INPA 20199 (1, $13.61 \mathrm{~mm} \mathrm{SL})$, rio Cuiuni, rio Negro basin. INPA 20200 (1, $18.67 \mathrm{~mm} \mathrm{SL})$, igarapé Aluini, rio Negro basin. INPA 21917 (13, 12.74-15.13 mm SL), same data as holotype. INPA 21918 (22 of 65, 11.83-22.02 mm SL), igarapé Juá Grande, lago Amanã, rio Japurá, rio Solimões basin. INPA 21928 (8, 10.92-14.04 m SL), rio Aiuanã, rio Negro basin. INPA 21929 (4, 14.51-17.01 mm SL), igarapé Zamula, rio Negro basin. INPA 21961 (3, 15.79-18.49 mm SL), igarapé Zamula, rio Negro basin. INPA 24527 (111, not measured), igarapé Paiol, rio Juma, rio Aripuanã, rio Madeira basin $\left(06^{\circ} 01^{\prime} 02^{\prime}\right.$ 'S, $\left.60^{\circ} 10^{\prime} 41^{\prime \prime} \mathrm{W}\right)$. INPA 24528 (15, not measured), rio Mariepauá, rio Madeira basin (05'22'16”'S, 60³5'58'W). INPA 24529 (18, not measured), igarapé Terra Preta, rio Atininga, rio Madeira basin,
Manicoré $\left(05^{\circ} 38^{\prime} 52^{\prime \prime} \mathrm{S}, 61^{\circ} 02^{\prime} 36^{\prime} \mathrm{W}\right)$. INPA 24530 (8, not measured), igarapé without name, rio Atininga, rio Madeira basin, Manicoré (0536'14'S, 61 ${ }^{\circ} 00^{\prime} 55^{\prime}$ 'W). MPEG 9719 (5, not measured), same data as INPA 21918. MZUSP 88067 (5, not measured), same data as INPA 21918. Pará: INPA 25030 (25, not measured), unnamed stream, Caxiuanã National Forest, Melgaço county ( $01^{\circ} 45^{\prime} 58^{\prime \prime}$ S, 51 $\left.1^{\circ} 30^{\prime} 34^{\prime \prime} \mathrm{W}\right)$. MPEG 6151 (2, 13.62-17.17 mm SL), rio Puraquequara, Caxiuanã National Forest. MPEG 7189 (60, not measured), same data as INPA 25030. MPEG 7190 (7, not measured), igarapé Curuá, Caxiuanã National Forest. MZUSP 12419 (2, 12.14-12.70 mm SL), lago Jacaré, rio Trombetas basin. MZUSP $15553(1,15.71 \mathrm{~mm} \mathrm{SL})$, lago Jacaré, rio Trombetas basin. MZUSP 21960 (8, 9.11-11.27 mm SL), rio Tapajós, island in front of Monte Cristo. Roraima: ANSP 180961 (9, not measured), INPA 20192 (1, 12.1mm SL), INPA 20195 (1, $13.53 \mathrm{~mm} \mathrm{SL}$ ), and INPA20196 (20 of 64, 10.35-

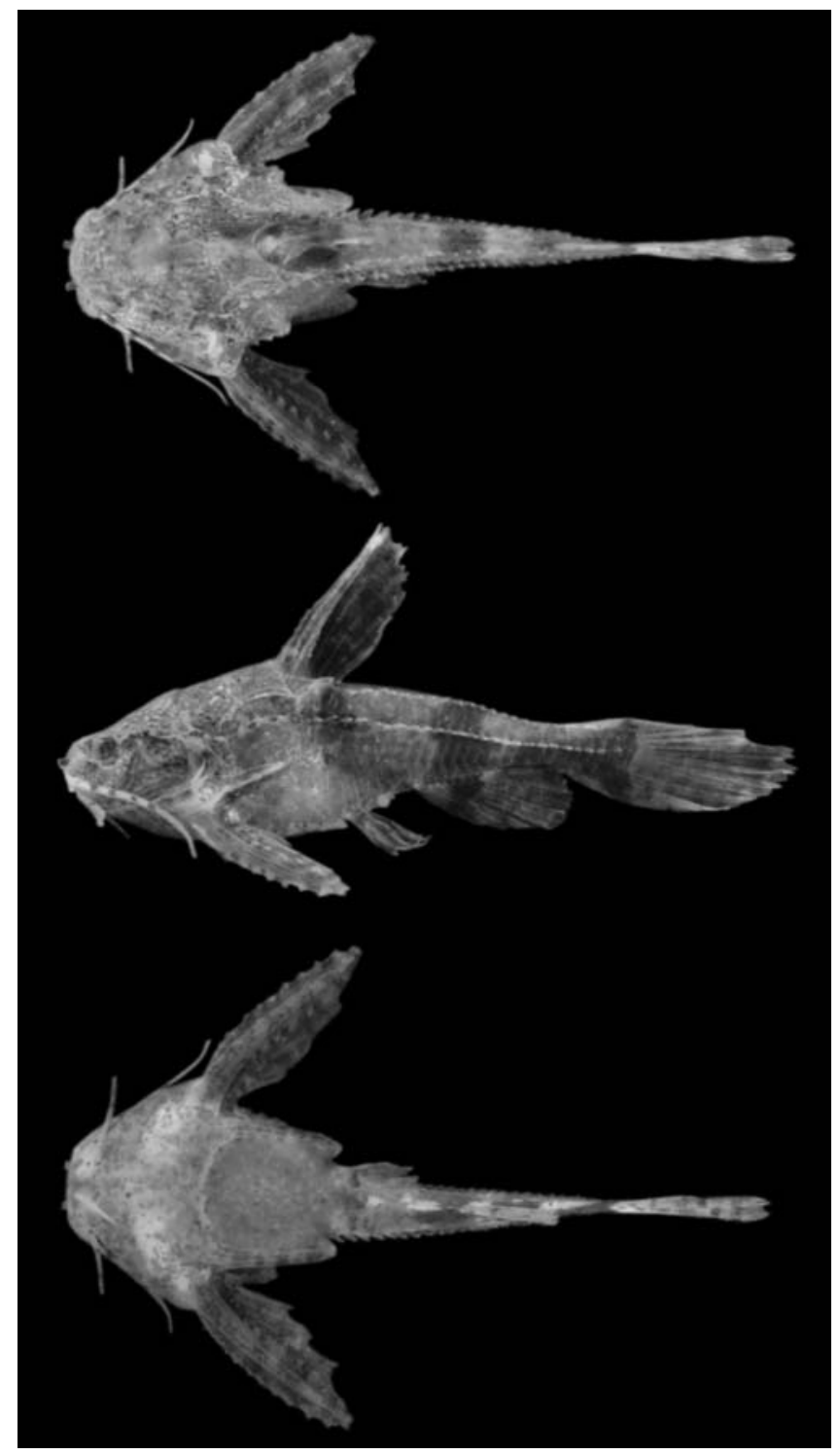

Fig. 5. Physopyxis ananas, holotype, INPA $24324(13.43 \mathrm{~mm}$ SL), rio Jutaí, rio Solimões basin, Amazonas State, Brazil. 
13.98 mm SL) (6 c\&s, not measured), igarapé Corumbaú, rio Branco, rio Negro basin. Guyana: ANSP 177255 (1, $15.93 \mathrm{~mm}$ SL), Siparuni River, Essequibo River basin (04\%4'14"N, $58^{\circ} 59^{\prime} 17^{\prime \prime} \mathrm{W}$ ). ANSP 177256 (13 of 20, 12.74-19.23 mm SL), Siparuni River, Essequibo River basin ( $04^{\circ} 48^{\prime} 59^{\prime \prime} \mathrm{N}$, 58 50'56”W). ANSP 177257 (7, 16.01-20.06 mm SL), same data as ANSP 177256. ANSP 177877 (1, $12.69 \mathrm{~mm} \mathrm{SL})$, Essequibo River (04'31'28'N, 58 33'37'W). ANSP 177918 (1, $12.25 \mathrm{~mm}$ SL), Burro burro River (0443'04'N, 58 51 '27'W). AUM 27814 (1, not measured), Essequibo River, Mazaruni-Potaro (05 $58^{\prime} 01^{\prime \prime} \mathrm{N}, 58^{\circ} 33^{\prime} 48^{\prime}$ 'W). AUM 27830 (4, not measured), Essequibo River, Mazaruni-Potaro (0559'30'N, 58 34'04'W). AUM 27849 (2, not measured), Essequibo River, MazaruniPotaro $\left(05^{\circ} 59^{\prime} 08^{\prime \prime} \mathrm{N}, 58^{\circ} 33^{\prime} 03^{\prime \prime} \mathrm{W}\right)$. INPA 25060 (5, not measured), same data as ANSP 177256. MZUSP 88068 (5, not measured), same data as ANSP 177256. Peru: Loreto: ANSP 116356 ( $1 \mathrm{c} \& s$, not measured), río Nanay, vicinity Iquitos. ANSP 116357 ( $1 \mathrm{c} \& s$, not measuerd), same data as ANSP 116356. ANSP $149336(5,11.52-13.59 \mathrm{~mm} \mathrm{SL})$, same data as ANSP 116356. ANSP 167599 (2, 17.01-18.76 mm SL), río Nanay (approx. 0353'S, 7327'W). ANSP 167600 (1, $17.55 \mathrm{~mm} \mathrm{SL}$ ), laguna Rocafuerte, río Nanay ( $03^{\circ} 46^{\prime}$ 'S, $\left.73^{\circ} 15^{\prime} \mathrm{W}\right)$. ANSP 167602 $(1,19.35 \mathrm{~mm} \mathrm{SL})$, same data as ANSP 167599. ANSP 177922 (1, $13.79 \mathrm{~mm}$ SL), río Nanay ( $\left.03^{\circ} 30^{\prime} \mathrm{S}, 7^{\circ} 15^{\prime} \mathrm{W}\right)$. ANSP 178208 (2, 14.40-18.42 mm SL), caño Santa Rita, río Nanay (0345’23”S,

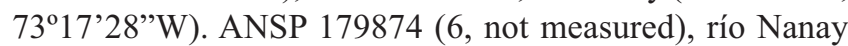
(035'21”S, 73³2'43”W). ANSP 180994 (3, not measured),

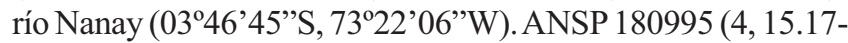
$20.35 \mathrm{~mm} \mathrm{SL}$ ), río Nanay (approx. $03^{\circ} 45^{\prime} \mathrm{S}, 7^{\circ} 17^{\prime} \mathrm{W}$ ). MUSM 26092 (3, not measured), same data as ANSP 180994. UNAP 315 (3, not measured), same data as ANSP 179874. Venezuela: Amazonas: ANSP $165816(1,10.61 \mathrm{~mm} \mathrm{SL})$, río Casiquiare (approx. 02 $48^{\circ} \mathrm{N} ; 65^{\circ} 58^{\prime} \mathrm{W}$ ).

Diagnosis. Physopyxis ananas can be distinguished from other Physopyxis species by presence of at least one additional row of spines on dorsal wings of lateral plates, above lateral line (Fig. 9). Additionally, P. ananas differs by having a more slender, slightly convergent and pointed coracoid processes $v s$. coracoid processes more robust with knob-like divergent tips in P. lyra. Physopyxis ananas also differs by having a complete series of lateral line plates $v s$. incomplete in P. cristata.

Description. Morphometric data summarized in Table 2. Largest specimen measures $22.02 \mathrm{~mm}$ SL. Body short and deep with greatest body depth at dorsal fin origin (body depth 26$37 \%$ of SL). Head and nuchal plate shaped and ornamented as in $P$. lyra. Eye anterior, orbital diameter slightly smaller than fontanel. Fontanel oval. Anterior nostril tubular and placed near border of snout. Posterior nostril nearer to orbit than to snout. Snout short, its length twice orbital diameter. Lacrimal bone well developed, dorsal and ventral margins serrated with conspicuous spines. Mouth terminal. Barbels simple, long, slightly compressed, with papillae along inferior surfaces. Maxillary barbel reaching to or surpassing tip of
Table 2. Descriptive morphometrics and counts of Physopyxis ananas $(\mathrm{N}=148) . \mathrm{SD}=$ Standard deviation. $\mathrm{Hol}=$ Holotype.

\begin{tabular}{|c|c|c|c|}
\hline & Hol & Range & Mean (SD) \\
\hline $\begin{array}{l}\text { Standard length }(\mathrm{mm}) \\
\end{array}$ & 13.43 & $9.11-22.02$ & \\
\hline Total length (mm) & 18.43 & $12.37-28.03$ & \\
\hline \multicolumn{4}{|c|}{ Percent of Standard length } \\
\hline Pre-dorsal length & 42.67 & $38.50-45.15$ & $41.98(1.44)$ \\
\hline Dorsal fin base & 13.03 & $10.79-17.59$ & $12.59(0.95)$ \\
\hline Body depth & 34.33 & $26.11-37.12$ & $31.37(2.01)$ \\
\hline Dorsal-adipose fin length & - & $21.48-32.29$ & $24.76(2.04)$ \\
\hline Adipose-caudal fin length & - & $6.97-17.20$ & $12.08(2.15)$ \\
\hline Pre-pectoral length & 30.53 & $26.86-34.86$ & $30.5(1.39)$ \\
\hline Pre-pelvic length & 54.58 & $45.15-61.25$ & $53.63(1.96)$ \\
\hline Pre-anal length & 66.27 & $58.53-$ & 65.61( \\
\hline Anal fin base & 16.53 & $13.58-26.13$ & 17.17 \\
\hline Caudal peduncle length & 11.02 & $5.94-13.70$ & 10.51( \\
\hline Caudal peduncle depth & 8.41 & $5.95-10.50$ & $8.41(0.77)$ \\
\hline Lateral plate depth & 14.3 & $2.26-17.81$ & $13.72(1.78)$ \\
\hline Superior lateral plate portion depth & 8.04 & $4.77-9.99$ & $7.72(0.91)$ \\
\hline Inferior lateral plate portion depth & 8.94 & $3.67-11.75$ & $8.4(1.82)$ \\
\hline Body depth at 4th plate & 17.8 & 14.3 & 17.65 \\
\hline spine length & 32.84 & 20.55 & 30.42 \\
\hline 1 spine length & 40.51 & $30.27-45.80$ & $39.3(2.84)$ \\
\hline Head length & 29.86 & $24.96-33.42$ & $28.88(1.53)$ \\
\hline Maxillary barbel length & 38.35 & $23.79-63.07$ & $36.97(8.06)$ \\
\hline Scapular girdle width & 44.53 & $37.08-48.83$ & $42.53(2.03)$ \\
\hline rocess length & 9.68 & 17.98 & $11.16(1.82)$ \\
\hline Corac & 23.31 & 17.8 & \\
\hline girdle mesial suture & 22.93 & $17.06-25.14$ & $21.92(1.42)$ \\
\hline Dist between coracoid process tips & 20.25 & $15.17-28.76$ & $20.18(2.39)$ \\
\hline Coracoid width & 28.44 & $21.30-35.27$ & $28.47(2.11)$ \\
\hline \multicolumn{4}{|c|}{ Percent of Head length } \\
\hline Head d & 102.49 & $83.23-115.32$ & $98.33(5.18)$ \\
\hline Orbi & 16.71 & $13.29-22.86$ & $17.97(1.94)$ \\
\hline Intero & 54.36 & $45.06-62.99$ & $53.13(3.05)$ \\
\hline Mouth inner width & 44.14 & $28.85-49.64$ & $41.18(3.62)$ \\
\hline \multirow[t]{2}{*}{ Snout length } & 32.67 & $26.36-42.64$ & $33.41(2.63)$ \\
\hline & Counts & & Mode \\
\hline Dorsal fin rays & $\mathrm{I}+5$ & $I+4-6$ & $\mathrm{I}+5$ \\
\hline Pectoral fin rays & $\mathrm{I}+4$ & $I+3-5$ & $\mathrm{I}+4$ \\
\hline Pelvic fin rays & 7 & & 7 \\
\hline Anal fin rays & 13 & $10-17$ & 13 \\
\hline Caudal fin rays & 12 & $9-12$ & 11 \\
\hline Dorsal spine serrae & 4 & $2-8$ & 4 \\
\hline Lateral plates & 25 & $23-27$ & 25 \\
\hline
\end{tabular}

postcleithral process. Outer mental barbel reaching point of insertion of pectoral spine. Length of inner mental barbel usually half length of outer mental barbel.

Scapular girdle and cleithrum as in P. lyra. Postcleithral process relatively short, its tip reaching to or slightly surpassing vertical through dorsal-spine origin. Coracoid processes very long, slender (larger than postcleithral process), with well defined straight longitudinal grooves and ridges along its entire length. Tip of coracoid process pointed, not expanded.

Dorsal and pectoral spines very strong; ornamented, shaped and serrated as in P. lyra.

Vast majority of observed specimens has 25 lateral plates (range 23-27, plates present in all specimens). Lateral plates with single medial to submedial row of posteriorly-directed spines and at least one additional row of spines on dorsal wings (Fig. 9). Lateral plates of both sides meeting dorsally in large specimens. Dorsal and ventral sections of lateral plates usually same size; in some specimens, dorsal wing smaller. 


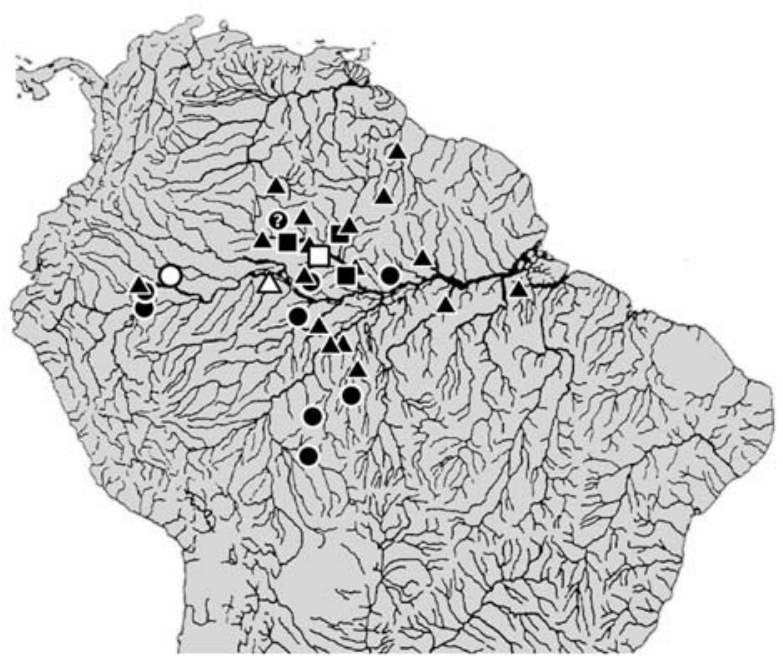

Fig. 6. Geographic distribution of Physopyxis lyra (circle), Physopyxis ananas (triangle), and Physopyxis cristata (squares). Open symbols are type localities. Record of $P$. lyra in rio Negro questionable.

Dorsal-fin rays I,5. Dorsal-fin origin situated at the anterior third of the body. Pectoral-fin rays I,4. Pectoral fin located at vertical through branchial opening. Pelvic-fin rays 7. Pelvic fin inserted at vertical through coracoid process tip, approximately at middle of body; tip of pelvic fin lightly surpassing anal-fin origin. Anal fin with 13 rays in total. Adipose fin absent in most specimens. When present, adipose fin small, membranous. Caudal fin truncate to slightly emarginate, with 8,3 or 7,4 rays.

Color in alcohol. Physopyxis ananas has a color pattern similar to that described for $P$. lyra, except that $P$. ananas is usually darker overall.

Distribution. Physopyxis ananas has the widest distribution among the species of the genus, occurring throughout lowlands in entire Amazon (including rio Negro) and rio Essequibo basins (Fig. 6).

Etymology. From the generic name for the pineapple, Ananas (Bromeliaceae). The specific name ananas is an allusion to stout body and spiny appearance of species due to presence of additional row(s) of spines on lateral plates.

Habitat. As mentioned for Physopyxis lyra, P. ananas can be found in shallow waters where organic debris accumulates, such as submerged litter banks. A large number of specimens were collected along with hundreds of specimens of $P$. lyra among the submerged roots of aquatic macrophytes (Paspallum repens, Poaceae) at lago Amanã, rio Japurá ba$\sin$. Physopyxis ananas and P. lyra also were collected together in rio Nanay (a moderate blackwater) upstream from Iquitos.

\section{Physopyxis cristata, new species}

Fig. 7

Holotype. INPA24325 (22.73 mm SL), mouth of igarapé Zamula, rio Negro, Amazonas State, Brazil (0051'48”'S, 6246’44”'W), N. L. Chao et al., 20 Oct 2002.

Paratypes (N= 23). Brazil: Amazonas: ANSP 180960 (2, 10.7$11.04 \mathrm{~mm} \mathrm{SL})$, same data as holotype. INPA 20193 (2, 13.38$14.42 \mathrm{~mm}$ SL), rio Caurés, rio Negro basin. INPA 20194 (1, $12.64 \mathrm{~mm} \mathrm{SL}$ ), rio Caurés, rio Negro basin. INPA 21915 (1, $10.64 \mathrm{~mm} \mathrm{SL})$, same data as holotype. INPA $21916(1,11.18 \mathrm{~mm}$ SL), igarapé Zamula, rio Negro basin. INPA $25063(1,10.98 \mathrm{~mm}$ SL), rio Cuiuni, rio Negro basin. MZUSP 76209 (2, not measured), beach of paraná do Jacaré, rio Negro basin. MZUSP 76210 (2, not measured), Urumari beach, two hours upstream Barcelos, rio Negro ( $\left.00^{\circ} 58^{\prime} \mathrm{S}, 62^{\circ} 57^{\prime} \mathrm{W}\right)$. MZUSP 76211 (1, not measured), Urumari beach, two hours upstream Barcelos, rio Negro (0058'S, 62 $\left.{ }^{\circ} 57^{\prime} \mathrm{W}\right)$. Roraima: INPA 25061 (1, $10.93 \mathrm{~mm}$ $\mathrm{SL})$, igarapé Corumbaú, rio Branco, rio Negro Basin. INPA $25062(7+2 \mathrm{c} \& \mathrm{~s}$, not measured), same data as INPA 25061.

Diagnosis. Physopyxis cristata can be easily distinguished from its congeners by possessing an incomplete lateral line that ranges between five and twelve lateral plates; lateral plates weakly ossified, externally visible only by posteriorly-oriented spine; tips of neural spines exposed, perforating skin middorsally along posterior portion of body, forming a crest between dorsal and caudal fins.

Description. Morphometric data summarized in Table 3. Largest specimen examined measures $22.73 \mathrm{~mm} \mathrm{SL}$. Body slightly slender, moderately deep with greatest body depth at origin of dorsal fin (body depth $27-30 \%$ of SL). Top of head and nuchal plates roof shaped, with bony ornamentation arranged in shallow grooves concentrated along nuchal shield crest and borders. Anterior nostril tubular and placed near border of snout. Posterior nostril nearer to orbit than to snout. Snout short, its length twice orbital diameter. Lacrimal bone well developed, dorsal and ventral margins serrated with conspicuous spines. Mouth terminal. Barbels simple, long, slightly compressed, with papillae along inferior surfaces. Maxillary barbel reaching to or surpassing tip of postcleithral process. Outer mental barbel reaching point of insertion of pectoral spine. Inner mental barbel slightly shorter than outer mental barbel.

Scapular girdle as in Physopyxis lyra. Cleithrum laterally expanded and visible from above, sculptured with shallow grooves up to postcleithral process. Postcleithral process relatively short, its tip reaching to or slightly surpassing vertical through dorsal-spine origin. Coracoid process very long, slender (larger than postcleithral process), with well defined straight longitudinal grooves and ridges along its entire length. Tip of coracoid process pointed, not expanded.

Dorsal and pectoral spines strongly ossified, serrated and grooved as in P. lyra.

Lateral line incomplete and asymmetric, ranging from five 
to 12 lateral plates. Lateral plates weakly developed and embedded beneath skin, with only retrorse spines visible from outside. Tips of neural spines of vertebrae visible externally, emerging middorsally in posterior portion of body, forming crest between dorsal and caudal fins. Some specimens with tips of vertebral hemal spines visible externally, emerging midventrally on caudal peduncle.

Dorsal-fin rays I,4 or I,5. Dorsal-fin origin situated at anterior third of body. Pectoral-fin rays I,4. Pectoral fin located at vertical through branchial opening. Pelvic-fin rays 7. Pelvic fin inserted at vertical through coracoid process tip, approximately at middle of body. Tip of pelvic-fin rays reaching to anal-fin origin. Anal fin with 15 rays. Adipose fin small, membranous. Caudal fin truncate to slightly emarginate, with 8,4 rays .

Color in alcohol. Physopyxis cristata has same color pattern as described for P. lyra.

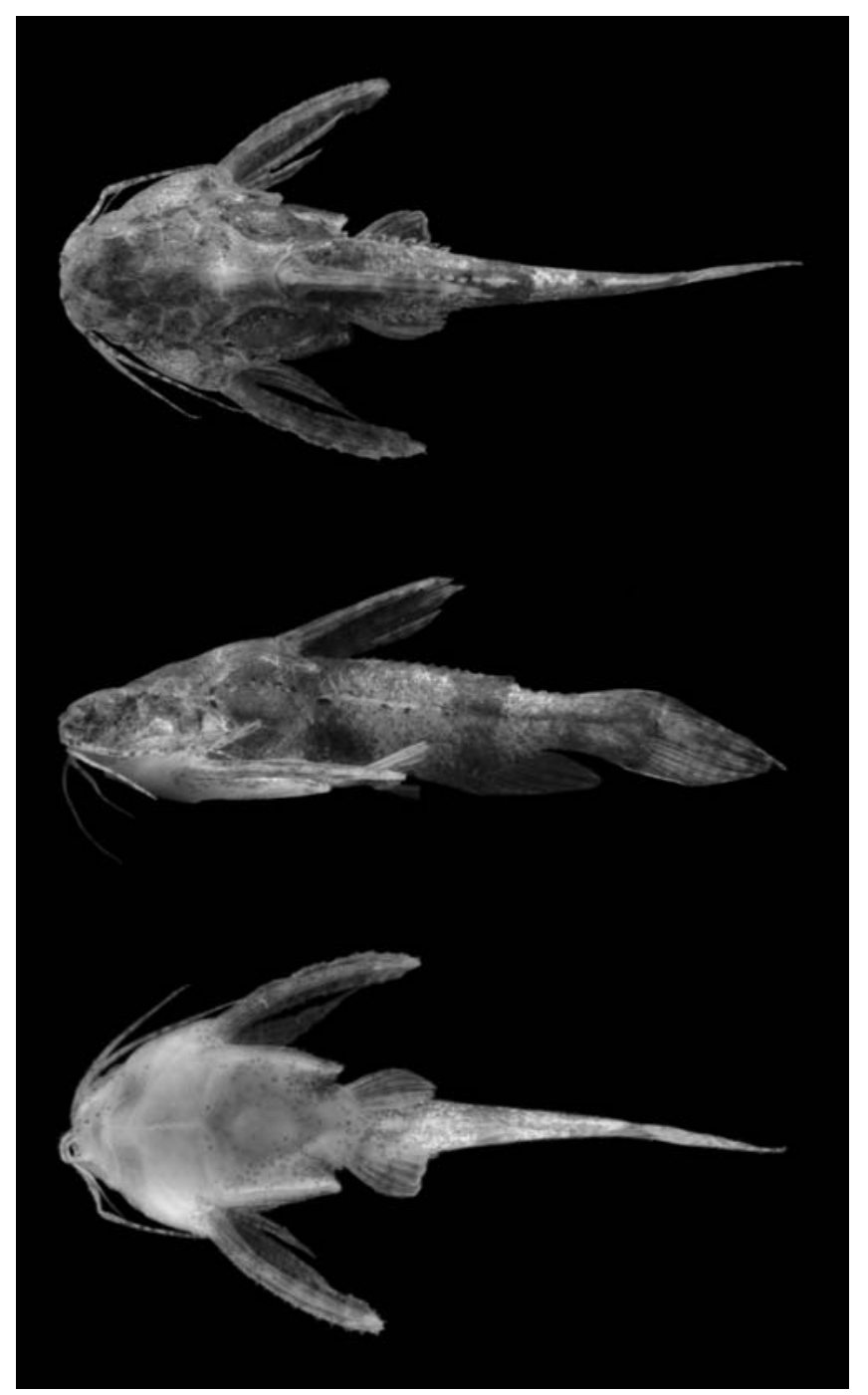

Fig. 7. Physopyxis cristata, holotype, INPA 24325 (22.73 mm $\mathrm{SL})$, mouth of igarapé Zamula, rio Negro, Amazonas State, Brazil.
Table 3. Descriptive morphometrics and counts of Physopyxis cristata $(\mathrm{N}=10) . \mathrm{SD}=$ Standard deviation. Hol = Holotype.

\begin{tabular}{|c|c|c|c|}
\hline & Hol & Range & Mean (SD) \\
\hline Standard length (mm) & 22.73 & $10.64-22.73$ & \\
\hline Total length (mm) & 30.17 & $13.51-30.17$ & \\
\hline \multicolumn{4}{|c|}{ Percent of Standard length } \\
\hline Pre-dorsal length & 40.65 & $39.40-41.11$ & $40.04(0.63)$ \\
\hline Dorsal fin base & 12.76 & $10.76-13.66$ & $12.6(0.99)$ \\
\hline Body depth & 28.68 & $27.17-30.14$ & $29.03(1.03)$ \\
\hline Dorsal-adipose fin length & 26 & $22.37-26.0$ & $23.98(1.22)$ \\
\hline Adipose-caudal fin length & 9.85 & $9.85-14.38$ & $11.54(1.52)$ \\
\hline Pre-pectoral length & 29.3 & $26.27-29.70$ & $28.52(1.08)$ \\
\hline Pre-pelvic length & 50.68 & $45.65-52.77$ & $49.93(2.41)$ \\
\hline Pre-anal length & 63.22 & $60.84-65.35$ & $62.97(1.51)$ \\
\hline Anal fin base & 22.88 & $22.72-25.04$ & $23.48(0.77)$ \\
\hline Caudal peduncle length & 7.83 & $4.92-8.46$ & $7.03(1.08)$ \\
\hline Caudal peduncle depth & 10.07 & $9.42-10.62$ & $10.06(0.37)$ \\
\hline Lateral plate depth & 4.14 & $2.35-4.14$ & $3.15(0.68)$ \\
\hline Superior lateral plate portion depth & - & - & - \\
\hline Inferior lateral plate portion depth & - & - & - \\
\hline Body depth at 4 th plate & - & - & - \\
\hline Dorsal spine length & 29.3 & $26.63-35.51$ & $31.22(3.39)$ \\
\hline Pectoral spine length & 40.61 & $34.57-41.48$ & $38.33(2.65)$ \\
\hline Head length & 28.2 & $26.64-29.27$ & $28.11(0.8)$ \\
\hline Maxillary barbel length & 42.89 & $29.07-42.89$ & $36.04(5.79)$ \\
\hline Scapular girdle width & 38.36 & $38.36-42.11$ & $39.6(1.29)$ \\
\hline Postcleithral process length & 8.8 & $5.93-10.19$ & $8.35(1.57)$ \\
\hline Coracoid process length & 21.21 & $19.66-22.84$ & $20.97(1.04)$ \\
\hline Pectoral girdle mesial suture & 18.52 & $17.71-20.02$ & $18.71(0.76)$ \\
\hline Dist between coracoid process tips & 22.44 & $16.49-22.44$ & $20.03(1.78)$ \\
\hline Coracoid width & 28.16 & $24.37-28.62$ & $26.81(1.54)$ \\
\hline \multicolumn{4}{|c|}{ Percent of Head length } \\
\hline Head depth & 88.3 & $85.10-96.34$ & $91.11(3.75)$ \\
\hline Orbital diameter & 13.42 & $13.42-25.16$ & $20.89(3.18)$ \\
\hline Interorbital width & 48.05 & $48.05-56.95$ & $54.01(3.18)$ \\
\hline Mouth inner width & 39.01 & $38.95-47.45$ & $42.57(2.99)$ \\
\hline \multirow[t]{2}{*}{ Snout length } & 35.41 & $32.12-38.38$ & $34.48(2.17)$ \\
\hline & Counts & & Mode \\
\hline Dorsal fin rays & $\mathrm{I}+5$ & $\mathrm{I}+4-5$ & $\mathrm{I}+5$ \\
\hline Pectoral fin rays & $\mathrm{I}+4$ & $I+3-4$ & $\mathrm{I}+4$ \\
\hline Pelvic fin rays & 7 & 7 & 7 \\
\hline Anal fin rays & 15 & $13-17$ & 15 \\
\hline Caudal fin rays & 11 & $10-12$ & 12 \\
\hline Dorsal spine serrae & 5 & $4-7$ & 5 \\
\hline Lateral plates & 10 & $5-12$ & 10 \\
\hline
\end{tabular}

Distribution. This species has been recorded only from middle portion of the rio Negro basin (Amazonas drainage), Amazonas State, Brazil.

Etymology. The name cristata, from Latin cristatus, meaning crested, in allusion to externally visible series of emergent neural spines.

Habitat. Some specimens included in type series were found in accumulated leaf litter, suggesting habitat similar to that observed in P. lyra. Dwarf cichlids of the genus Apistogramma and juvenile specimens of Amblydoras were collected with specimens of $P$. cristata.

\section{Discussion}

Higuchi (1992) stated that the basal condition among doradids is a well-developed pectoral girdle, with the postcleithral process much larger than the coracoid process. 
In Physopyxis, the cleithrum is large but the coracoid process is extremely long, strong and exposed (i.e., much larger than the postcleithral process). The coracoid process is proportionally longer in P. lyra than in P. ananas and P. cristata and shows longitudinal grooves and ridges in all three species. In P. lyra, these longitudinal grooves and ridges turn abruptly to the sides at the end of the coracoid process, following the enlargement of this extremity (see description for details). In the other two species the grooves and ridges run parallel to the longitudinal axis of the body along the entire coracoid
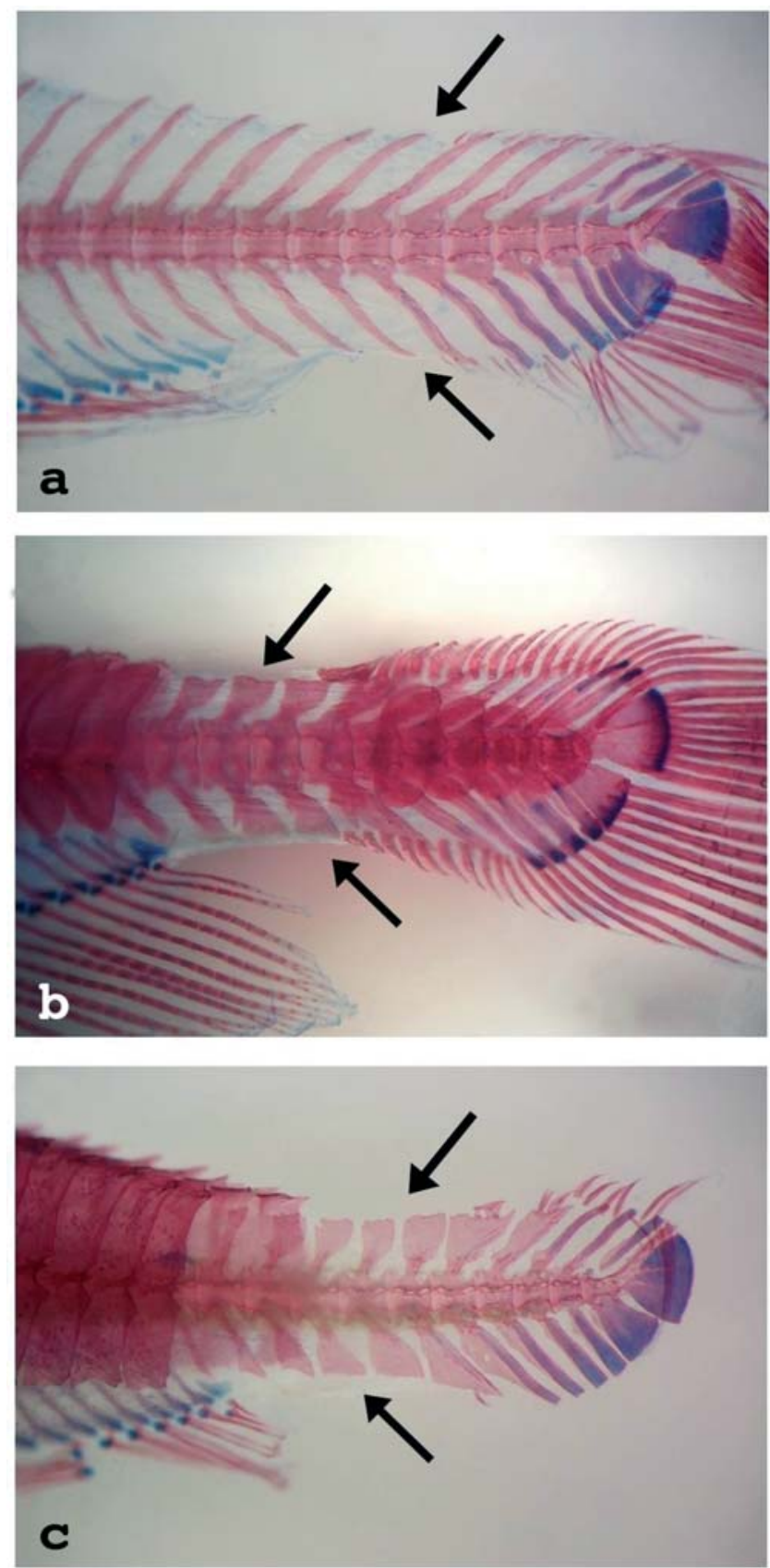

Fig. 8. Lateral view of caudal fin skeleton and caudal peduncle vertebrae of Physopyxis: a. P. cristata (INPA 25062, $10.99 \mathrm{~mm} \mathrm{SL}$ ); b. P. lyra (INPA 20321, $25.54 \mathrm{~mm} \mathrm{SL}$ ); and c. $P$. ananas (INPA 20196, $13.05 \mathrm{~mm} \mathrm{SL}$ ). process. Interestingly, even in juveniles of $P$. lyra which do not yet show the development of the distal enlargement of the coracoid process, the grooves and ridges are already directed laterally near the tip, forming little spines on the border of the bone.

Coracoid process enlargement shows geographic variation. Specimens of $P$. lyra from the rio Guaporé, for instance, do not show the enlargement on the tip of the process, but do have the juvenile groove and ridge pattern of specimens from other drainages (i.e., grooves and ridges directed laterally near tip of coracoid process).

Higuchi (1992) pointed out a unique feature of the fin rays of Physopyxis: a terminal bifurcation of the flexible fin-rays (Fig. 8) (vs. non-terminal bifurcation of rays). This feature was observed in all examined specimens of the three species. Fin-ray counts show a great deal of variation, especially for anal and caudal fins (Tables 1-3). Distal bifurcation in flexible fin-rays may cause miscounts due to difficulty in correctly interpreting the limits between branched and unbranched rays. The caudal fin in Physopyxis also is distinctive. The primitive condition for Siluriformes is the presence of nine principal caudal-fin rays (i.e., rays articulating with parhypural and hypurals only) on both upper and lower lobe. However, most catfish groups, including doradids, exhibit more principal rays on the lower lobe (e.g., 7+8, 7+9, 8+9, etc.) (Lundberg $\&$ Baskin, 1969). Physopyxis differs by having fewer principal rays in the lower caudal fin lobe than in the upper lobe. In most cases there are eight principal rays on the upper lobe and only four on the lower lobe (Fig. 8).

The most plastic feature in Physopyxis is the lateral plate complex, varying from a complete lateral line covered by welldeveloped plates, running along the entire body (e.g., most adult $P$. lyra and $P$. ananas), to an incomplete lateral line, with small and poorly ossified plates (e.g., adult $P$. cristata). Few doradids show lateral plate reduction or interruption (e.g., Hassar, Nemadoras leporhinus, Doraops, Kalyptodoras, and Wertheimeria, sensu Sabaj, 2002). However, the only doradid previously reported to have an incomplete lateral line is an undescribed genus and species related to Amblydoras (Higuchi, 1992; Britski et al., 1999). The lateral plate pattern found in Physopyxis cristata is similar to the one found in Higuchi's undescribed taxon. In Higuchi's cladistic analysis of the family, a clade formed by Amblydoras and Higuchi's undescribed genus is the sister group of Physopyxis. A more complete phylogenetic study is needed to clarify the level of homology of lateral line reduction among doradids.

In Physopyxis lyra and $P$. ananas (species with complete lateral lines), there is substantial size-related variation in the degree of development, ossification and ornamentation of the lateral plates. Young specimens have undeveloped plates that do not meet dorsally. Increase in body size is accompanied by plate development until complete middorsal contact is made between the plates on both sides of the body. The direction of plate development is cephalo-caudal, whereby the posterior plates are the last ones to develop completely and contact dorsally. In Physopyxis ananas, an additional 
row of plate spines is formed early in development and becomes larger and more conspicuous with age. This kind of development generally is not seen in other spiny catfishes (e.g., Loricariidae), in which the young exhibit large spines that become less conspicuous with increased size. The number of additional spines and the body size at which full spine development is attained vary with locality or drainage. Similar size specimens from different locations may exhibit distinct stages of plate ornamentation. The variation in plate ornamentation found in specimens of $P$. ananas can be seen in Fig. 9.

Lateral plate ornamentation in $P$. lyra also may vary with drainage. Specimens from the rio Uatumã have the posterior border of the lateral plates serrated, mainly dorsally. Specimens from the rio Guaporé may have an incomplete lateral line but exhibit all other diagnostic features of $P$. lyra. In a group of 38 specimens from this drainage, 21 have 24 lateral

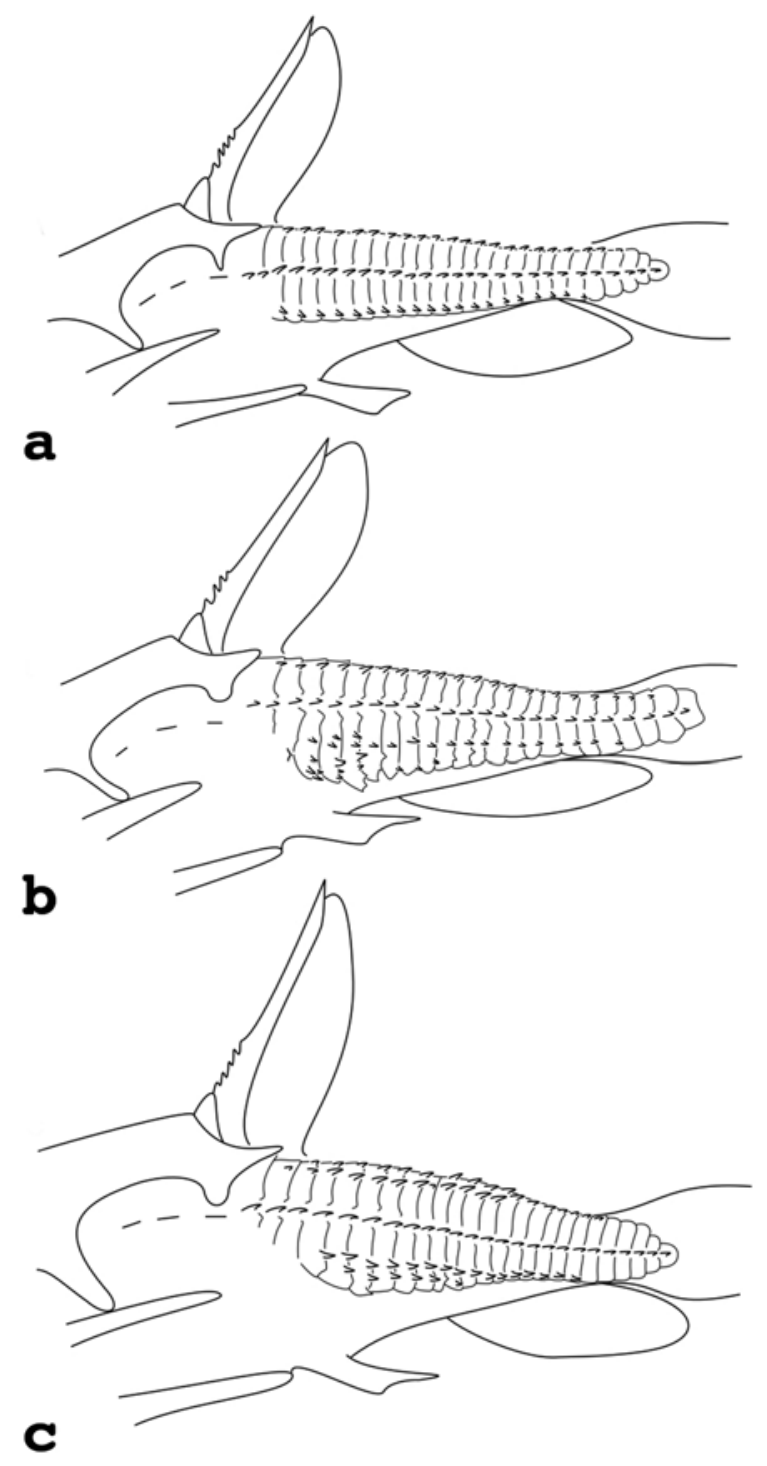

Fig. 9. Physopyxis ananas. Diagram of three different types of lateral plate ornamentation: a. MPEG 7189 Caxiuanã, 15.21 mm SL; b. ANSP 177256. Essequibo River, 17.50 mm SL; c. MPEG 7189, Caxiuanã, 16.53 mm SL. plates or less, and four small specimens (12.62-16.37 mm SL) are apparently naked. One of these specimens has an inconspicuous ossification along the lateral line, suggesting an early stage in plate development.

Physopyxis cristata is the only doradid known to have an externally visible extension of the neural spines on pre-caudal and caudal vertebrae (see description for more details). Higuchi (1992) cited as an autapomorphy for P. lyra, monotypic at that time, the presence of flattened and enlarged neural and hemal spines. Physopyxis ananas has the same feature. Physopyxis cristata, however, has the slender condition of neural and hemal spines that is found in other doradids (Fig. 8).

Physopyxis also is characterized by the lack of an anterior nuchal plate. Higuchi (1992) noted that the anterior nuchal plate might be fused to the middle nuchal plate, based on the typical shapes of these plates in other doradids. This character was first noticed in some Auchenipteridae by Ferraris (1988), and might be homoplastic within Doradoidea. In the Doradidae, some taxa of Doradini have the anterior nuchal plate greatly reduced, grain shaped (Higuchi, 1992: 122, character 84). This is clearly not homologous to the condition presented in Physopyxis, in which the anterior nuchal plate appears fused to the middle nuchal plate.

The large sample of Physopyxis examined resulted in the expansion of the geographic distribuition of the genus and the recognition of intra and interespecific variation. However, intrageneric and intrafamilial phylogenetic relationships remain largely unresolved.

\section{Acknowledgements}

We greatly benefitted from comments on the manuscript by Jansen Zuanon (INPA), José Birindelli (MZUSP) and Mark Sabaj (ANSP). We acknowledge the following curators for loans of specimens: Jonathan Armbruster (AUM), John Lundberg (ANSP), Osvaldo Oyakawa (MZUSP), and Wolmar Wosiacki (MPEG). Mark Sabaj (ANSP) provided the pictures of the holotype of Physopyxis lyra. The All Catfish Species Inventory (NSF DEB-0315963) granted funds to LMS to visit the MZUSP fish collection. We also acknowledge INPA Graduate School Program and CAPES (MEC, Brazil) for a Masters scholarship for LMS. Material from rio Madeira and rio Aripuanã were made available through fieldwork funded by the PROBIO Project (MMA, Brazil/GEF, World Bank). We thank Michel Catarino (Mamirauá Sustainable Developing Institute/MCT), Gislene Torrente Villara, Odirlene Marinho Ribeiro and many others who collaborated in the field.

\section{Literature Cited}

Britski, H. A., K. Z. S. Silomon \& B. S. Lopes. 1999. Peixes do pantanal. Manual de identificação. Brazil: Empresa Brasileira de Pesquisa Agropecuária, Brasilia. 184 p.

Burgess, W. E. 1989. An Atlas of Freshwater and Marine Catfishes. A preliminary survey of the Siluriformes. T.F.H. Publications, Neptune City. 784 p. 
Cope, E. D. 1871. Fishes from the Amazon above the mouth of Rio Negro. Proceedings of the Academy of Natural Sciences of Philadelphia, 23: 55.

Cope, E. D. 1872. On the Fishes of the Ambyacu River. Proceedings of the Academy of Natural Sciences of Philadelphia, 23: 250-294.

Eigenmann, C. H. 1925. A review of the Doradidae, a family of South American Nematognathi, or cat-fishes. Transactions of the American Philosophical Society, 22(5): 280-365.

Ferraris, C. 1988. The Auchenipteridae putative monophyly and systematics with a classification of the neotropical doradoid catfishes (Ostariophysi, Siluriformes). Unpublished Ph.D. Thesis, City University of New York, New York.

Higuchi, H. 1992. A phylogeny of the South American thorny catfishes (Osteichthyes; Siluriformes; Doradidae). Unpublished Ph.D. Thesis, Harvard University, Cambridge.

Higuchi H., H. A. Britski \& J. C. Garavello. 1990. Kalyptodoras bahiensis, a new genus and species of thorny catfishes from northeastern Brazil (Siluriformes: Doradidae). Ichthyological Exploration of Freshwaters, 1(3): 219-225.

Jaeger, E. C. 1950. A source-book of biological names and terms (2 ed.). C. C. Thomas, Springfield. 323 p.
Leviton, A. E., R. H. Gibbs Jr., E. Heal \& C. E. Dawson. 1985. Standards in herpetology and ichthyology. Part I. Standard symbolic codes for institutional resource collections in herpetology and ichthyology. Copeia 1985(3): 802-832.

Lundberg, J. G. \& J. N. Baskin. 1969. The caudal skeleton of the catfishes, order Siluriformes. American Museum Novitates, 2398: 1-49.

Sabaj, M. H. 2002. Taxonomy of the Neotropical thorny catfishes (Siluriformes: Doradidae) and revision of genus Leptodoras. Unpublished Ph.D. Thesis, University of Illinois, Urbana.

Sabaj, M. H. \& C. J. Ferraris Jr. 2003. Family Doradidae. Pp. 456-469 In: R. E. Reis, S. O. Kullander \& C. J. Ferraris Jr. (Eds.). Check list of the freshwater fishes of South and Central America. Edipucrs, Porto Alegre. 729p.

Taylor, W. R. \& G. C. Dyke. 1985. Revised procedures for staining and clearing small fishes and other vertebrates for bone and cartilage study. Cybium, 4(2): 108-118.

Received October 2005 Accepted November 2005 